four to five days in the untreated case and about a day less in those treated. All patients alive on arrival at a treatment centre should recover if given appropriate fluids sufficiently quickly and in sufficient quantity.

There are two phases of treatment-the correction of dehydration and electrolyte imbalance, and the maintenance of this correction. Intravenous fluid (the 5/4/1 Dacca solution) of the following composition serves for both: $\mathrm{NaCl}_{5} \mathrm{~g}$., $\mathrm{NaHCO}_{3} 4 \mathrm{~g}$., $\mathrm{KCl} 1 \mathrm{~g}$., distilled water to 1 litre. ${ }^{7}$

In the correction phase the patients are severely dehydrated and acidotic, but it is sufficient to determine the degree of dehydration alone by a simple procedure such as the measurement of plasma specific gravity (P.S.G.) by the copper sulphate drop method of Phillips and colleagues. ${ }^{8}$ The amount of intravenous fluid required can then be calculated according to the formula (Estimated P.S.G. minus 1.025 ) $\times 200,000=\mathrm{ml}$. fluid required. For example, the estimated P.S.G. is 1.037 . Subtraction of 1.025 leaves 0.012 . Multiplication of this by 200,000 gives 2,400 , and this is the required amount of fluid in ml. The fluid must be given intravenously as fast as is possible, usually within half an hour or less.

When polyethylene catheters are available for intravenous use, it is convenient to insert one for infusion, but the administration of fluid must not be delayed through waiting for the catheter: infusion should be started through a needle and a change made later if necessary. A particular advantage of the polyethylene catheter is that it may be used for measuring the central venous pressure (C.V.P.), and this in turn may be used to assess the adequacy of fluid replacement instead of the plasma specific gravity. After approximately 1 litre of the infusion fluid has been given as rapidly as possible the patient is propped up in bed with the upper half of the body at $45^{\circ}$ from the horizontal. The drip is then stopped and disconnected from the infusion set, and the polyethylene tube is held vertically alongside the thorax. The fluid in it will level off at the central venous pressure. If the pressure is more than $5 \mathrm{~cm}$. below the sternal angle, further fluid should be infused until the pressure is $1-2 \mathrm{~cm}$. below the sternal angle. At this point the hydration can be assumed to be adequate, and further infusion of fluid is regulated to balance losses as indicated below. Fast infusion of fluid is safe so long as the central venous pressure does not rise above the sternal angle. Above this level there is a danger of inducing pulmonary oedema.

Maintenance therapy is simple. The stool volume should be measured hourly and the rate of infusion of the same mixture regulated to equal it. Vomiting almost invariably ceases with the passage of the first few watery stools, and the patient should be given water and glucose-containing drinks ad libitum plus $1-2 \mathrm{~g}$. tetracycline per day. ${ }^{9}$

In countries where the disease may occur in epidemic proportions the very large amounts of intravenous fluids required ( 20 or more litres per patient) may put a strain on supplies. It is therefore important to know that recently a regimen of treatment by mouth has been developed at the

British Medical fournal, 1970, 3, 601

2 C. M. O. Circular 13/70.

Mosley, W. H., et. al., Bulletin of the World Health Organization, 1969, 40, 187 - Mosley, W. H., et al., Bulletin of the World Health Organization, 1969,
40,187.

- Phillips, R. A., Federation Procecdings, 1964, 23, 705.

- Rahaman, M. M., Rahman, W., Hare, W. K., Hare, R. D., and Phillips, R. A. Eighth International Congress of Tropical Medicine, Abstracts and Reviews, 1968, p. 490.

Nalin, R., et al., Lancet, 1968, 2, 370.

* Phillips, R. A., et al., Fournal of Biological Chemistry, 1950, 183, 305

- Pierce, N. F., et al., British Medical fournal, 1968, 3, 277.

- Cash, R. A., Nalin, D. R., Forrest, J. N., and Abrutyn, E., Lancet, 1970, 2, 549

$"$ P.S.C.R.L. Technical Committee Report, 1969.
Cholera Research Laboratories established under the joint auspices of the South-east Asia Treaty Organization and Pakistan. It may be used in the maintenance phase of treatment with substantial saving of intravenous fluid and may even be used alone in mild cases. ${ }^{10}$ The success of this regimen depends on the fact that glucose given by mouth enhances absorption of sodium. Glycine even further enhances absorption of sodium, and treatment incorporating this as well as glucose and electrolytes in the fluid has been under trial in the same laboratories, with encouraging results. ${ }^{11}$ It is here fitting to pay a tribute to Dr. R. A. Phillips and his team at those laboratories, for they have made substantial contributions to the understanding of this disease and its management.

\section{Renal Glomerular Disease}

Similar symptoms can arise from a variety of pathological changes in the renal glomeruli. It is not surprising, therefore, that earlier classifications of glomerulonephritis-such as that of A. Ellis ${ }^{1}$ - which were based on clinical and necropsy studies, lost their relevance when the use of renal biopsy enabled the structure of the kidneys to be examined during life. A complete diagnosis in kidney disease requires a description of the clinical syndrome, of the renal lesions, and, when known, of the cause of the condition. But correlations between clinical conditions and pathological lesions are now well enough known to allow us to divide primary glomerular disease on a pathological basis into several categories. These are not necessarily discrete entities, but each is sufficiently homogeneous to be useful in determining the prognosis for individual patients and in providing a guide to treatment.

Such a classification has been summarized recently by J. Churg, R. Habib, and R. H. R. White. ${ }^{2}$ They described five major groups-minimal change, focal glomerulosclerosis, membranous (epimembranous) nephropathy, proliferative glomerulonephritis, and chronic glomerulonephritis. The last group represents the end stage of many conditions, and glomerular destruction is too advanced for the recognition of specific features.

Proliferative glomerulonephritis comprises an assortment of pathological conditions and has five subdivisions. These are: the diffuse exudative lesion, characteristic of the acute phase of post-streptococcal glomerulonephritis; mesangial proliferation, which is seen in subsiding post-streptococcal glomerulonephritis but which also occurs without evidence of previous streptococcal infection; focal proliferation, often associated with the Henoch-Schonlein syndrome or other multi-system diseases; epithelial crescents, which, when large, cause obliteration of glomeruli, leading to rapidly progressive renal failure; and membranoproliferative glomerulonephritis. The renal lesions of multi-system diseases, such as systemic lupus erythematosus, polyarteritis nodosa, subacute bacterial endocarditis, and Goodpasture's syndrome, may be indistinguishable from those of primary proliferative glomerulonephritis. Most of the categories are familiar, but in two cases-focal glomerulosclerosis and membranoproliferative glomerulonephritis-the associations between clinical and pathological features have only recently been clarified.

In focal glomerulosclerosis (found in about $9^{\circ} \prime$, of children with the nephrotic syndrome ${ }^{2} 3$ ) totally or segmentally sclerosed glomeruli are interspersed with normal glomeruli, the juxtamedullary glomeruli being involved earliest. ${ }^{4}$ The condition may be difficult to distinguish on renal biopsy from 
minimal change in the kidney. But their separation is essential because focal glomerulosclerosis is a progressive condition leading ultimately to death from renal failure and because most of the patients with this type of disease do not respond to prednisone. It is reasonable to speculate that some patients said to have a steroid-resistant minimal-change nephrotic syndrome may in fact have focal glomerulosclerosis.

Membranoproliferative glomerulonephritis is reviewed in detail by Drs. J. S. Cameron, E. F. Glasgow, C. S. Ogg, and R. H. R. White in an article at page 7 of this issue of the B.M.F. They found sufficient uniformity in the clinical, histological, and immunological features of 50 patients to separate it from other forms of proliferative glomerulonephritis. The characteristic glomerular abnormality was a combination of mesangial proliferation and capillary-wall thickening. Lobulation of the glomerular tuft was accentuated in miny cases by an increase of fibrillary material as well as by cellular proliferation in the lobular stalks. The capillarywall thickening, which was diffuse, was caused by deposition of argyrophilic fibrils between the endothelial cytoplasm and the basement membrane and by increase in thickness of the basement membrane itself.

Similar appearances have been described before and have been designated by various terms such as chronic lobular or mixed membranous and proliferative glomerulonephritis. When proliferation is inconspicuous, the histological appearance may resemble that of membranous nephropathy, but the presence of subepithelial projections from the basement membrane in silver-impregnated thin sections enables the latter condition to be distinguished.

Persistently low serum concentrations of $\beta_{1}$ c globulin (the C3 component of complement) were found in patients with this type of renal histology by C. D. West and his colleagues, ${ }^{5}$ who used the designation of persistent hypocomplementaemic nephritis. Cameron and his colleagues prefer to rely on the morphological criteria for diagnosis, for though most of their patients had deficiency of complement at some stage of their illness $16 \%$ did not. But measurement of serum C3 concentration is of value in the differential diagnosis of nephritis, since the only other renal diseases in which it is low-active nephritis in lupus erythematosus and the acute phase of poststreptococcal glomerulonephritis-are easily differentiated on other grounds. The cause of the hypocomplementaemia in membranoproliferative glomerulonephritis is at present uncertain, as is the aetiology of the nephritis. Only a minority of patients have evidence of preceding streptococcal infection.

The patients were mostly children of school age and young adults, with a small preponderance of females. In common with other types of glomerulonephritis the membranoproliferative form does not produce a specific functional disturbance, and various presenting features were seen. The nephrotic syndrome was the most frequent. In series of patients with the nephrotic syndrome membranoproliferative glomerulonephritis accounts for about $6 \%$ of the cases, both in children ${ }^{23}$ and in adults. ${ }^{6}$ The other modes of presentation were the acute nephritic syndrome, symptomless proteinuria, and visible haematuria. Heavy proteinuria and microscopical or macroscopic haematuria were found in virtually all patients and impairment of renal function and hypertension in several.

1 Ellis, A., Lancet, 1942, 1, 1.

2 Churg, J., Habib, R., and White, R. H. R., Lancet, 1970, 1, 1299.

3 White, R. H. R., Glasgow, E. F., and Mills, R. J., Lancet, 1970, 1, 1353.

4 Rich, A. R., Bulletin of the fohns Hopkins Hospital, 1957, 100, 173.

West, C. D., McAdams, A. J., McConville, J. M., Davis, N. C., and Holland, N. H., Fournal of Pediatrics, 1965, 67, 1089.

- Sharpstone, P., Ogg, C. S., and Cameron, J. S., British Medical fournal, $1969,2,533$.
The natural course of the disease was not firmly established, but generally it seemed to be slowly progressive, with the development of end-stage renal failure some years after onset. But three patients died within a year of presentation, and half of the patients followed up showed no deterioration of renal function during the period of observation. Treatment with corticosteroid or cytotoxic drugs did not appear to produce any benefit.

\section{Athletes' Deaths}

Sudden death in sport may be due to physical injury, either external or internal, to disease, or to circulatory or metabolic stresses beyond the tolerable limits for the athlete. The environment and the possible hazards are carefully controlled in most sports, though in the more active and violent competitions a careful balance must be maintained between risk of injury and personal achievement. Human failure in the form of inadequate training, carelessness, or ill health is the main cause of most accidents. Less frequent, though nevertheless important, is failure of equipment.

In a session on "death in sport" at the 18th World Congress of Sports Medicine held in Oxford from 6-11 September most of the papers were concerned with the effect of physical stresses on the cardiovascular system. Professor Ernst Jokl, of Kentucky University, presented case histories to support his belief that potentially fatal cardiac disease may not be manifest until the terminal collapse. Post-mortem examinations had shown that these conditions fell into four groups: firstly, coronary athero- and arteriosclerosis with myocardial disease; secondly, a congenital abnormality of the coronary arteries; thirdly, chronic myocarditis; and, fourthly, a cardiac tumour. Dr. Nina Graevskaya, from the First Sports Medicine Dispensary of Moscow, thought that the most frequent cause of athletes' deaths was underlying disease, but the irregular imposition of very great work loads could precipitate cardiac failure in persons who had not been fully conditioned to such stresses, and this view reflected the general feeling of the meeting. Many speakers drew attention to the danger of exercise during or soon after respiratory infections, when a transitory myocarditis may occur. Dr. Kocnar, of Czechoslovakia, recommended an electrocardiographic test before returning to sport after common infections of the respiratory system as of great prophylactic importance. In Japan, according to one speaker, most deaths among junior high school students were caused by drowning in swimming exercises during school hours, whereas most deaths among senior students resulted from contusion of the brain during extracurricular judo.

At no time was there any doubt that as a contribution to health and physical well-being the value of sport greatly outweighs any potential risk to life. Indeed Professor S. E. Strauzenberg, director of a rehabilitation centre in the German Democratic Republic, emphasized the value of graded exercises after myocardial infarction. The special hazards of boxing, however, were discussed in a report from the Royal College of Physicians of London last year. ${ }^{1}$ Abstracts of papers presented at the conference have been published by the British Association of Sport and Medicine, who were the hosts of the congress.

\footnotetext{
Royal College of Physicians Committee on Boxing, Report on the Medical
} Aspects of Boxing. Royal College of Physicians of London, 1969. 